\title{
A Fifth Order Alternative Mapped WENO Scheme for Nonlinear Hyperbolic Conservation Laws
}

\author{
Uttam Singh Rajput and Krishna Mohan Singh* \\ Department of Mechanical and Industrial Engineering, Indian Institute of Technology \\ Roorkee, Roorkee 247667, India
}

Received 20 October 2020; Accepted (in revised version) 25 February 2021

\begin{abstract}
In this work, we have developed a fifth-order alternative mapped weighted essentially nonoscillatory (AWENO-M) finite volume scheme using non-linear weights of mapped WENO reconstruction scheme of Henrick et al. (J. Comput. Phys., 207 (2005), pp. 542-567) for solving hyperbolic conservation laws. The reconstruction of numerical flux is done using primitive variables instead of conservative variables. The present scheme results in less spurious oscillations near discontinuities and shows higher-order accuracy at critical points compared to the alternative WENO scheme (AWENO) based on traditional non-linear weights of Jiang and Shu (J. Comput. Phys., 228 (1996), pp. 202-228). The third-order Runge-Kutta method has been used for solution advancement in time. The Harten-Lax-van Leer-Contact (HLLC) shock-capturing method is used to provide necessary upwinding into the solution. The performance of the present scheme is evaluated in terms of accuracy, computational cost, and resolution of discontinuities by using various one and two-dimensional test cases.
\end{abstract}

AMS subject classifications: 35L65, 65M08

Key words: High resolution scheme, unsteady, non-linear weights, numerical fluxes, alternative WENO scheme, hyperbolic equations.

\section{Introduction}

The development of high-order spatial accurate schemes for the solution of nonlinear hyperbolic equations is of great interest for the last few decades. The solution of hyperbolic equations contains discontinuities like rarefaction, shock wave, contact surfaces, and shear lines. These discontinuities are always present in the solution. Therefore, we need a high order scheme to capture these discontinuities without spurious oscillations. Weighted essentially non-oscillatory (WENO) and essentially non-oscillatory

*Corresponding author.

Emails: krishna.singh@me.iitr.ac.in (K. Singh), urajput@me.iitr.ac.in (U. Rajput) 
(ENO) schemes are widely used higher-order shock-capturing methods for solving nonlinear hyperbolic equations [1]. In the ENO scheme, the reconstruction of the convective flux is based on a fixed candidate stencil. In contrast, in the WENO scheme, reconstruction of the convective flux is done by the dynamic combination of different ENO stencils [2]. Harten et al. [3,4] developed a high order ENO finite volume scheme, which is a modified version of the total-variation diminishing (TVD) scheme in [5]. Casper and Atkins [6], presented a high order non-oscillatory scheme for two-dimensional problems. Liu et al. [7] introduced a third-order WENO finite volume scheme. Jiang and Shu [8] constructed a 5th order WENO scheme denoted by WENO-JS. Later, Balsara and Shu [9] developed a very high-resolution WENO scheme (up to 11th order) in their work. WENO-JS can be made a finite volume or finite difference based scheme. The finite volume schemes are flexible and robust compared to the finite difference schemes [10]. Several finite volume WENO schemes have been designed and applied to curvilinear grid [11,12], non-uniform grids [13], unstructured grids [14] and structured grids [15-17]. The alternative WENO scheme developed by Shu and Osher [18] is an efficient scheme in the finite difference framework. It has been used with Cartesian [19] as well as curvilinear grids [20]. Liu [21] conducted a comparative study for the fifth-order alternative WENO scheme using different approximate Riemann solver for inviscid cases. The alternative methodology has also been used with the compact-WENO scheme [22], and the Hermite WENO scheme [23]. The alternative WENO approach could not perform with optimal accuracy at the points where derivatives become zero (critical points). Henrick et al. [24] designed the fifth-order mapped WENO scheme (WENO-M), in which mapping of nonlinear weights results in achieving 5th order accuracy at the critical points. Later WENO-Z scheme $[25,26]$ was developed, which provides better resolution and desirable convergence rate at the critical points. Recently, Wang et al. [27] studied an alternative finite difference WENO scheme with WENO-Z weights on a structured grid. Gao et al. [28] have extended the alternative WENO scheme framework to seventh and ninth order. This methodology has also been applied to shallow water equations, and multicomponent flows [29-31]. In the present work, we have designed a fifth-order alternative mapped WENO finite volume scheme using WENO-M nonlinear weights [24] for solving nonlinear hyperbolic equations. The proposed scheme is denoted by AWENO$\mathrm{M}$. It evaluates numerical flux using the reconstruction of primitive variables (solution variable) rather than conservative variables. The WENO reconstruction procedure based on conservative variables produces spurious oscillations as compared to primitive variables $[32,33]$. The developed scheme results in small numerical oscillations near discontinuities (shock waves and contact surfaces) and gives the optimal rate of convergence at critical points. The shock-capturing HLLC method [34,35] has been adopted for splitting the numerical flux due to its robustness. The performance of the resultant scheme is analyzed through different one and two-dimensional test problems. We assess the robustness of the proposed AWENO-M scheme by comparing computational cost and resolution of discontinuities with the traditional WENO scheme (WENO-JS) [8], WENOM scheme [24], and the alternative WENO scheme based on WENO-JS nonlinear weights 
(AWENO-JS) [19].

The present work is described as follows: we give a general framework of the physical problem for two dimensions in Section 2. In Section 3, we provide a detailed description of the alternative mapped WENO scheme for the evaluation of numerical flux. In Section 4, we have presented numerical results of one and two-dimensional problems. Concluding remarks are discussed in Section 5.

\section{General framework}

The non-linear hyperbolic equation in a conservative form is given by

$$
\frac{\partial \mathbf{U}}{\partial t}+\frac{\partial \mathbf{G}}{\partial x_{1}}+\frac{\partial \mathbf{H}}{\partial x_{2}}=0
$$

where

$$
\mathbf{U}=\left[\begin{array}{c}
\rho \\
\rho u_{1} \\
\rho u_{2} \\
E
\end{array}\right], \quad \mathbf{G}=\left[\begin{array}{c}
\rho u_{1} \\
\rho u_{1}^{2}+p \\
\rho u_{1} u_{2} \\
u_{1}(E+p)
\end{array}\right], \quad \mathbf{H}=\left[\begin{array}{c}
\rho u_{2} \\
\rho u_{2} u_{1} \\
\rho u_{2}^{2}+p \\
u_{2}(E+p)
\end{array}\right] .
$$

Here, $\mathbf{U}$ is the vector of conservative variables, $\rho, p$, and $E$ are density, pressure, and total energy respectively. $u_{1}$ and $u_{2}$ are the velocities in coordinate directions $x_{1}$ and $x_{2}$. G and $\mathbf{H}$ are numerical (convective) fluxes. The semi-discretized form of (2.1) is given by

$$
\frac{d \mathbf{U}_{i j}}{d t}=-\frac{1}{\Delta x_{1}}\left(\mathbf{G}_{i+\frac{1}{2}, j}-\mathbf{G}_{i-\frac{1}{2}, j}\right)-\frac{1}{\Delta x_{2}}\left(\mathbf{H}_{i, j+\frac{1}{2}}-\mathbf{H}_{i, j-\frac{1}{2}}\right)=\mathbf{L}_{i j},
$$

where $\mathbf{U}_{i j}$ is the conservative variable at the $(i, j)$ th point, $\Delta x_{1}$ and $\Delta x_{2}$ are the grid spacing in $x_{1}$ and $x_{2}$ directions. $\mathbf{G}_{i \mp 1 / 2, j}$ and $\mathbf{H}_{i, j \mp 1 / 2}$ are the numerical fluxes in $x_{1}$ and $x_{2}$ direction, at the cell interfaces $x_{1_{i \mp 1 / 2, j}}$ and $x_{2_{i, j \neq 1 / 2}}$, respectively. The numerical fluxes at the cell boundaries are evaluated using fifth order alternative mapped WENO scheme discussed in next section. We solve (2.2) using 3rd order TVD Runge-Kutta method [36] as given below

$$
\left\{\begin{array}{l}
\mathbf{U}_{i j}^{0}=\mathbf{U}_{i j}^{n}+\Delta t \mathbf{L}_{i j}\left(\mathbf{U}^{n}\right), \\
\mathbf{U}_{i j}^{1}=(3 / 4) \mathbf{U}_{i j}^{n}+(1 / 4) \mathbf{U}_{i j}^{0}+(1 / 4) \Delta t \mathbf{L}_{i j}\left(\mathbf{U}^{0}\right), \\
\mathbf{U}_{i j}^{n+1}=(1 / 3) \mathbf{U}_{i j}^{n}+(2 / 3) \mathbf{U}_{i j}^{1}+(2 / 3) \Delta t \mathbf{L}_{i j}\left(\mathbf{U}^{1}\right) .
\end{array}\right.
$$

\section{Alternative mapped WENO scheme (AWENO-M)}

We consider the one-dimensional form of the Euler equations to describe an alternative mapped WENO scheme for the calculation of numerical flux. The conservative form of the 1-D Euler equation is

$$
\frac{d \mathbf{v}}{d t}+\frac{d \mathbf{f}(\mathbf{v})}{d x}=0,
$$


in which, $\mathbf{v}=[\rho, \rho u, E]^{T}$ is the conservative variable and $\mathbf{f}=\left[\rho u, \rho u^{2}+p, u(E+p)\right]^{T}$ is the numerical flux. Here, $E=0.5 \times \rho\left(u^{2}\right)+p /(\gamma-1)$ is the total energy per unit volume and $\gamma=1.4$ is specific heat ratio. The fully discretized form of (3.1) can be written as

$$
\mathbf{v}^{n+1}=\mathbf{v}^{n}-\frac{\Delta t}{\Delta x}\left(\overline{\mathbf{f}}_{i+\frac{1}{2}}-\overline{\mathbf{f}}_{i-\frac{1}{2}}\right),
$$

where $\overline{\mathbf{f}}_{i+1 / 2}$ is the approximated value of alternative numerical flux normal to the cell interface $x_{i+1 / 2}$. An alternative form proposed in [18] for the evaluation of approximated numerical flux $\overline{\mathbf{f}}_{i+1 / 2}$ is given by

$$
\overline{\mathbf{f}}_{i+\frac{1}{2}}=\mathbf{f}_{i+\frac{1}{2}}+\sum_{k=1}^{p-1} b_{2 k} \Delta x^{2 k}\left(\frac{\partial^{2 k} \mathbf{f}}{\partial x^{2 k}}\right)_{i+\frac{1}{2}}+\mathcal{O}\left(\Delta x^{2 p-1}\right),
$$

where $\mathbf{f}_{i+1 / 2}=\mathbf{f}\left(\mathbf{v}_{i+1 / 2}\right)$ is the numerical flux calculated through fifth-order AWENO-M reconstruction procedure as discussed in this section. The coefficients $b_{2 k}$ can be evaluated through the Taylor series expansion. For example, the approximated numerical flux for $p=3$ is given by

$$
\overline{\mathbf{f}}_{i+\frac{1}{2}}=\mathbf{f}_{i+\frac{1}{2}}-\frac{1}{24} \Delta x^{2}\left(\frac{\partial^{2} \mathbf{f}}{\partial x^{2}}\right)_{i+\frac{1}{2}}+\frac{7}{5760} \Delta x^{4}\left(\frac{\partial^{4} \mathbf{f}}{\partial x^{4}}\right)_{i+\frac{1}{2}} .
$$

The last two terms of (3.4) are approximated by using the central difference scheme [22] as

$$
\left\{\begin{array}{l}
\left(\frac{\partial^{2} \mathbf{f}}{\partial x^{2}}\right)_{i+\frac{1}{2}}=\left(1 / 48 \Delta x^{2}\right)\left(-5 \mathbf{f}_{i+3}+39 \mathbf{f}_{i+2}-34 \mathbf{f}_{i+1}-34 \mathbf{f}_{i}+39 \mathbf{f}_{i-1}-5 \mathbf{f}_{i-2}\right), \\
\left(\frac{\partial^{4} \mathbf{f}}{\partial x^{4}}\right)_{i+\frac{1}{2}}=\left(1 / 2 \Delta x_{1}^{4}\right)\left(\mathbf{f}_{i+3}-3 \mathbf{f}_{i+2}+2 \mathbf{f}_{i+1}+2 \mathbf{f}_{i}-3 \mathbf{f}_{i-1}+\mathbf{f}_{i-2}\right) .
\end{array}\right.
$$

\subsection{Shock capturing method}

To capture the discontinuities, we need a shock-capturing method which provides necessary upwinding into the solution. The shock-capturing method has been used to split the numerical flux $\mathbf{f}_{i+1 / 2}$ into the left $\left(\mathbf{f}^{-}\right)$and right $\left(\mathbf{f}^{+}\right)$states using left wave $\left(\mathbf{v}^{-}\right)$and right wave $\left(\mathbf{v}^{+}\right)$configurations respectively. The HLLC method [35] is a three wave configuration model, i.e., left $\left(\mathbf{v}^{-}\right)$, right $\left(\mathbf{v}^{+}\right)$, and intermediate (or star) state $\left(\mathbf{v}_{*}\right)$. The intermediate state is further divided into two star states, i.e., $\mathbf{v}_{*}^{-}$and $\mathbf{v}_{*}^{+}$using contact discontinuity $s_{*}$. The procedure is as follows:

$$
\mathbf{v}_{i+\frac{1}{2}}= \begin{cases}\mathbf{v}^{-}, & s^{-}>0 \\ \mathbf{v}_{*}^{-}, & s^{-} \leq 0<s_{*} \\ \mathbf{v}_{*}^{+}, & s_{*} \leq 0<s^{+} \\ \mathbf{v}^{+}, & s^{+}<0\end{cases}
$$


where $s^{-}$and $s^{+}$are the left and right state wave speed [37]. The intermediate state $\mathbf{v}_{*}^{ \pm}$is given by

$$
\mathbf{v}_{*}^{ \pm}=\rho^{ \pm}\left(\frac{s^{ \pm}-u^{ \pm}}{s^{ \pm}-s_{*}}\right)\left[\begin{array}{c}
1 \\
s_{*} \\
\frac{E^{ \pm}}{\rho^{ \pm}}+\left(s_{*}-u^{ \pm}\right)\left(s_{*}+\frac{p^{ \pm}}{\rho^{ \pm}\left(s^{ \pm}-u^{ \pm}\right)}\right)
\end{array}\right],
$$

where $s_{*}$ is given by

$$
s_{*}=\frac{\left(\rho^{-} u^{-} s^{-}-\rho^{-} u^{-} u^{-}\right)-\left(\rho^{+} u^{+} s^{+}-\rho^{+} u^{+} u^{+}\right)+p^{+}-p^{-}}{\rho^{+} u^{+}-\rho^{+} s^{+}-\rho^{-} u^{-}+\rho^{-} s^{-}} .
$$

The numerical flux $\mathbf{f}_{i+1 / 2}$ at the cell boundary is given by

$$
\mathbf{f}_{i+\frac{1}{2}}= \begin{cases}\mathbf{f}^{-}, & s^{-}>0 \\ \mathbf{f}_{*}^{-}=\mathbf{f}^{-} s^{-}\left(\mathbf{v}_{*}^{-}-\mathbf{v}^{-}\right), & s^{-} \leq 0<s_{*}, \\ \mathbf{f}_{*}^{+}=\mathbf{f}^{+} s^{+}\left(\mathbf{v}_{*}^{+}-\mathbf{v}^{+}\right), & s_{*} \leq 0<s^{+} \\ \mathbf{f}^{+}, & s^{+}<0\end{cases}
$$

The spatial accuracy of the numerical flux (3.8) is first-order accurate. To develop high order shock-capturing method, we need to reconstruct the left $\left(\mathbf{f}^{-}\right)$and the right $\left(\mathbf{f}^{+}\right)$state of numerical flux using WENO reconstruction [1].

\subsection{WENO reconstruction}

To describe the reconstruction procedure, let's consider a smooth scalar function $v(x)$. The left reconstructed value $v_{i+1 / 2}^{-}$of function $v(x)$ is given by

$$
v_{i+1 / 2}^{-}=\omega_{0}^{t} v_{0}+\omega_{1}^{t} v_{1}+\omega_{2}^{t} v_{2}
$$

where $v_{k}$ are the interpolated values of $k$ th candidate stencil $(k=0,1,2)$ as given below

$$
\left\{\begin{array}{l}
v_{0}=1.8334 v_{i}-1.667 v_{i-1}+0.334 v_{i-2}, \\
v_{1}=0.334 v_{i+1}+0.8334 v_{i}-0.1667 v_{i-1}, \\
v_{2}=-0.1667 v_{i+2}+0.8334 v_{i+1}+0.334 v_{i},
\end{array}\right.
$$

and $\omega_{k}^{t}(k=0,1,2)$ are the traditional non-linear weights defined by Jiang and Shu [8] as

$$
\omega_{k}^{t}=\frac{a_{k}^{t}}{\sum_{0}^{2} a_{k}^{t}}, \quad a_{k}^{t}=\frac{d_{k}}{\left(\epsilon+\beta_{k}\right)^{2}} .
$$

Here, $d_{k}$ are ideal weight coefficients $\left(d_{0}=1 / 10, d_{1}=3 / 5\right.$ and $\left.d_{2}=3 / 10\right)$ and $\epsilon$ is a small constant value to avoid zero denominator. The smoothness indicators $\beta_{k}$ are

$$
\left\{\begin{array}{l}
\beta_{0}=\left(1.084 v_{i}-2.168 v_{i-1}+1.084 v_{i-2}\right)^{2}+\left(0.75 v_{i}-v_{i-1}+0.25 v_{i-2}\right)^{2} \\
\beta_{1}=\left(1.084 v_{i+1}-2.168 v_{i}+1.084 v_{i-1}\right)^{2}+\left(-0.25 v_{i+1}+0.25 v_{i-1}\right)^{2}, \\
\beta_{2}=\left(1.084 v_{i+2}-2.168 v_{i+1}+1.084 v_{i}\right)^{2}+\left(0.25 v_{i+2}-v_{i+1}+0.75 v_{i}\right)^{2} .
\end{array}\right.
$$


WENO scheme based on traditional non-linear weights $\omega_{k}^{t}$ is called WENO-JS scheme. It loses its fifth-order rate of convergence at critical points. To resolve this issue, Henrick et al. [24] constructed a WENO-M scheme, in which non-linear weights are mapped by mapping functions $g_{k}(\omega)$ to attain optimal convergence at critical points. The mapping functions $g_{k}(\omega)(k=0,1,2)$ are given by

$$
g_{k}(\omega)=\frac{\omega\left(d_{k}^{2}+\omega^{2}+d_{k}-3 d_{k} \omega\right)}{\omega\left(1-2 d_{k}\right)+d_{k}^{2}} .
$$

The mapping function $g_{k}(\omega)$ has the following properties: $g_{k}(0)=g_{k}^{\prime}\left(d_{k}\right)=g_{k}^{\prime \prime}\left(d_{k}\right)=0$, $g_{k}\left(d_{k}\right)=d_{k}$, and $g_{k}(1)=1$. The mapped non-linear weights $\omega_{k}^{m}(k=0,1,2)$ designed in [24] are given by

$$
\omega_{k}^{m}=\frac{a_{k}^{m}}{\sum_{0}^{2} a_{k}^{m}}, \quad a_{k}^{m}=g_{k}\left(\omega_{k}^{t}\right)
$$

The left reconstructed value $v_{i+1 / 2}^{-}$for the WENO-M scheme, using mapped non-linear weights $\omega_{k}^{m}$, can be written as

$$
v_{i+1 / 2}^{-}=\omega_{0}^{m} v_{0}+\omega_{1}^{m} v_{1}+\omega_{2}^{m} v_{2}
$$

The right reconstructed value $v_{i+1 / 2}^{+}$for WENO-JS or WENO-M scheme can be easily evaluated through symmetry.

\subsection{AWENO-M reconstruction}

The AWENO-M scheme evaluates the numerical flux (3.8) using WENO-M weights (3.14). In this scheme, the reconstruction of the left $\left(\mathbf{f}^{-}\right)$and the right $\left(\mathbf{f}^{+}\right)$state of numerical flux is calculated by using primitive variables $(u$ and $p)$, considering that conservative variable components ( $\rho u$ and $E$ ) produce spurious oscillations near discontinuities as compared to primitive variables $[32,33]$.

\section{Numerical results}

We evaluate the performance of the present AWENO-M scheme for solving different one and two-dimensional test problems. We compare the computational cost (CPU time), resolution, and convergence rate of the AWENO-M scheme with the WENO-JS, WENO$\mathrm{M}$, AWENO-JS, and exact solutions. In this section, AWENO-JS and AWENO-M schemes are together called AWENO schemes, and WENO-JS and WENO-M are together called WENO schemes. The convergence rate, $r_{c}$, is calculated by

$$
r_{c}=\frac{\log \left(\text { error }_{\Delta x} / \text { error }_{\Delta x / 2}\right)}{\log 2} .
$$




\subsection{Convergence test at critical point}

For test of convergence of WENO and AWENO schemes, we consider the error in computation of derivative $(d g / d x)$ of the function $g(x)=x^{3}+\cos (x)$ at the critical point $x=0$ [24]. Results obtained with $\epsilon=10^{-40}$ are summarised in Table 1 . These results clearly show that the WENO-JS and AWENO-JS schemes are 3rd order accurate, and the WENO-M scheme is approximately fifth-order accurate. In contrast, the proposed AWENO-M scheme results in less dissipation and optimal order of accuracy (fifth-order).

Table 1: The $L^{\infty}$ error and $r_{c}$ of WENO and AWENO schemes for calculation of $d g / d x$ function near a critical point $(x=0)$ with $\epsilon=10^{-40}$.

\begin{tabular}{||c|cccccccc||}
\hline \multirow{2}{*}{$\Delta \mathrm{x}$} & \multicolumn{2}{|c}{ WENO-JS } & \multicolumn{2}{c|}{ WENO-M } & \multicolumn{2}{c||}{ AWENO-JS } & \multicolumn{2}{c|}{ AWENO-M } \\
\cline { 2 - 9 } & $L^{\infty}$ & $r_{c}$ & $L^{\infty}$ & $r_{c}$ & $L^{\infty}$ & $r_{c}$ & $L^{\infty}$ & $r_{c}$ \\
\hline 0.001 & $1.6 e-9$ & - & $6.1 e-14$ & - & $3.1 e-11$ & - & $4.2 e-14$ & - \\
0.0005 & $2.1 e-10$ & 2.96 & $2.1 e-15$ & 4.86 & $3.3 e-12$ & 3.24 & $1.3 e-15$ & 4.99 \\
0.00025 & $2.7 e-11$ & 2.98 & $6.9 e-17$ & 4.94 & $3.2 e-13$ & 3.39 & $4.2 e-17$ & 4.98 \\
0.000125 & $3.4 e-12$ & 2.99 & $2.2 e-18$ & 4.97 & $3.0 e-14$ & 3.42 & $1.3 e-18$ & 4.99 \\
0.0000625 & $4.2 e-13$ & 2.99 & $7.0 e-20$ & 4.98 & $2.7 e-15$ & 3.49 & $4.1 e-20$ & 4.99 \\
\hline
\end{tabular}

\subsection{Linear advection test case}

The one-dimensional linear advection equation can be written as

$$
v_{t}+v_{x}=0 \text {. }
$$

Initial conditions $(t=0)$ are

$$
v_{0}(x)= \begin{cases}{[0.67 g(x, a)+0.167 g(x, a-b)+0.167 g(x, a+b)],} & x \in[-0.80,-0.60], \\ 1.0, & x \in[-0.40,-0.20], \\ 1.0-|10.0 x-1.0|, & x \in[0.00,0.20], \\ {[0.67 h(x, c)+0.167 h(x, c-b)+0.167 h(x, c+b)],} & x \in[0.40,0.60], \\ 0.0, & \text { otherwise, }\end{cases}
$$

where $a=-0.7, b=0.005, c=0.5, g(x, a)=e^{-\phi(x-a)^{2}}, \phi=(\log 2) / 36 b^{2}, h(x, c)=$ $\sqrt{\left.\max \left(1-\beta^{2}(x-c)^{2}\right), 0\right)}$, and $\beta=10$. Eq. (4.2) is solved up to time $t=8$ for domain $[-1,1]$ with $N=300$ grid points. Table 2 and Fig. 1 show that the AWENO-M scheme captures sharp discontinuities more accurately. Further, it is also more accurate and less dissipative in comparison to AWENO-JS and WENO schemes.

\subsection{One dimensional accuracy test}

We consider one dimensional Euler equations for testing the accuracy of high order schemes. The initial $(t=0)$ condition is $(p, u, \rho)=(1.0,1.0,1.0+0.2 \sin (\pi x))$. Computations have been performed up to time $t=2.0$ with CFL number 0.9 for domain $[0.0,2.0]$. 


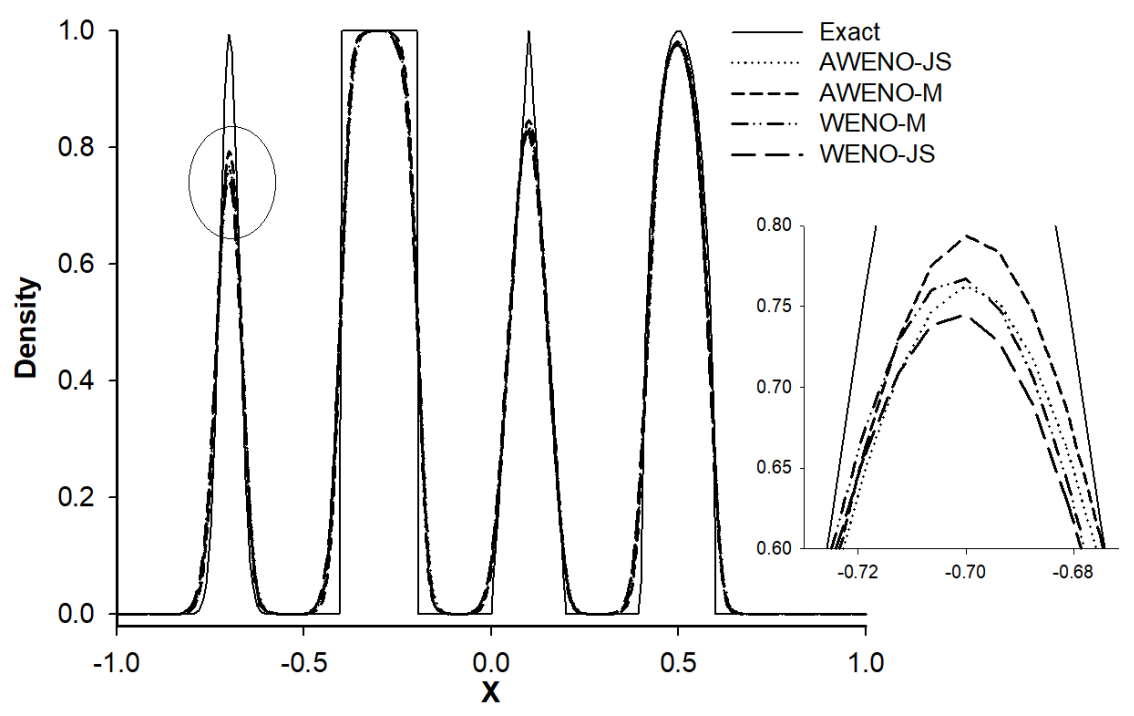

Figure 1: Simulation results of high order schemes (WENO and AWENO) for the linear advection problem with $N=300$ and $t=8$.

Table 2: The $L_{1}$ norm and $r_{c}$ of WENO and AWENO schemes, for linear advection equation.

\begin{tabular}{||c|cccccccc||}
\hline \multirow{2}{*}{$N$} & \multicolumn{2}{|c}{ WENO-JS } & \multicolumn{2}{c|}{ WENO-M } & \multicolumn{2}{c|}{ AWENO-JS } & \multicolumn{2}{c||}{ AWENO-M } \\
\cline { 2 - 9 } & $L_{1}$ & $r_{\mathcal{C}}$ & $L_{1}$ & $r_{\mathcal{C}}$ & $L_{1}$ & $r_{\mathcal{C}}$ & $L_{1}$ & $r_{\mathcal{C}}$ \\
\hline 40 & $4.9 e-1$ & - & $3.7 e-1$ & - & $4.2 e-1$ & - & $3.1 e-1$ & - \\
80 & $2.6 e-1$ & 0.90 & $2.0 e-1$ & 0.89 & $2.3 e-1$ & 0.89 & $1.6 e-1$ & 0.93 \\
160 & $1.2 e-1$ & 1.07 & $9.1 e-2$ & 1.13 & $1.1 e-1$ & 1.04 & $7.5 e-2$ & 1.12 \\
320 & $5.1 e-2$ & 1.28 & $3.7 e-2$ & 1.29 & $4.6 e-2$ & 1.25 & $3.0 e-2$ & 1.31 \\
640 & $2.4 e-2$ & 1.10 & $1.7 e-2$ & 1.11 & $2.2 e-2$ & 1.11 & $1.4 e-2$ & 1.16 \\
1280 & $1.2 e-2$ & 0.98 & $8.7 e-3$ & 0.98 & $1.1 e-2$ & 0.98 & $6.7 e-3$ & 1.01 \\
2560 & $6.2 e-3$ & 0.96 & $4.4 e-3$ & 0.97 & $5.6 e-3$ & 0.98 & $3.4 e-3$ & 0.99 \\
\hline
\end{tabular}

The exact solution for density is evaluated using $\rho(x, t)=1.0+0.2 \sin (\pi x-\pi t)$. Table 3 shows the $L_{1}$ error norm and rate of convergence $r_{c}$ for density. We can remark that all schemes show fifth order accuracy. The $L_{1}$ errors evaluated using proposed AWENO-M scheme are relatively smaller than the AWENO-JS and WENO schemes. Thus, AWENO$\mathrm{M}$ is more accurate than the other three schemes.

\subsection{One dimensional Euler problems}

Here, we solve the isolated contact discontinuity, 123 problem, shock-density wave interaction, Lax problem, shock-entropy wave interaction, and Sod's test. Computations have been performed using $\epsilon=10^{-40}$ for AWENO-M and WENO-M scheme, and $\epsilon=10^{-6}$ for AWENO-JS and WENO-JS scheme. We have used initial conditions on pressure $p$, veloc- 
Table 3: The $L_{1}$ norm and $r_{c}$ of one dimensional euler equation by the WENO and AWENO schemes.

\begin{tabular}{||c|cccccccc||}
\hline \multirow{2}{*}{$N$} & \multicolumn{2}{|c}{ WENO-JS } & \multicolumn{2}{c}{ WENO-M } & \multicolumn{2}{c|}{ AWENO-JS } & \multicolumn{2}{c||}{ AWENO-M } \\
\cline { 2 - 9 } & $L_{1}$ & $r_{c}$ & $L_{1}$ & $r_{c}$ & $L_{1}$ & $r_{c}$ & $L_{1}$ & $r_{c}$ \\
\hline 10 & $2.9 e-3$ & - & $6.5 e-4$ & - & $9.4 e-4$ & - & $3.0 e-4$ & - \\
20 & $8.8 e-5$ & 5.04 & $1.9 e-5$ & 5.10 & $2.8 e-5$ & 5.05 & $8.1 e-6$ & 5.23 \\
40 & $2.7 e-6$ & 5.02 & $5.6 e-7$ & 5.08 & $8.7 e-7$ & 5.03 & $2.2 e-7$ & 5.21 \\
80 & $8.5 e-8$ & 5.01 & $1.7 e-8$ & 5.04 & $2.7 e-8$ & 5.01 & $6.3 e-9$ & 5.11 \\
160 & $2.6 e-9$ & 5.00 & $5.2 e-10$ & 5.01 & $8.3 e-10$ & 5.01 & $1.9 e-10$ & 5.07 \\
320 & $8.2 e-11$ & 5.00 & $1.6 e-11$ & 5.00 & $2.6 e-11$ & 5.00 & $5.8 e-12$ & 5.02 \\
640 & $2.6 e-12$ & 5.00 & $5.1 e-13$ & 5.00 & $8.1 e-13$ & 5.00 & $1.8 e-13$ & 5.00 \\
1280 & $8.0 e-14$ & 5.00 & $1.6 e-14$ & 5.00 & $2.5 e-14$ & 5.00 & $5.6 e-15$ & 5.00 \\
\hline
\end{tabular}

ity $u$, and density $\rho$ to obtain a numerical solution with $N$ grid points.

\subsubsection{Isolated contact discontinuity}

The left and right states of the pressure, velocity, and density field for isolated contact discontinuity are $[p, u, \rho]_{l}=(1,0.1,1.4)$ and $[p, u, \rho]_{l}=(1,0.1,1)$. Computation has been performed up to time $t=2$ with CFL number 0.9 for $N=150$ grid points. Results with the proposed AWENO-M scheme based on the primitive variable reconstruction and conservative variable reconstruction are shown in Fig. 2. Similar density profiles are obtained with the primitive and the conservative variable reconstructions. However, the conservative variable reconstruction produces spurious oscillations in the velocity profile (Fig. 2(b)). A possible reason for spurious oscillations is that the characteristic speeds (or eigenvalues) that separate the left and right state in any flux splitting method are dependent on primitive variables rather than conservative variables. Therefore, we have used primitive variables for reconstructing the left and right state numerical flux (3.8) in this work.

\subsubsection{3 problem}

The left and right states of pressure, velocity, and density field for the 123 problem are $[p, u, \rho]_{l}=(0.4,-2,1), x \in[0,0.5)$ and $[p, u, \rho]_{r}=(0.4,2,1), x \in[0.5,1)$ respectively. We evaluate solution up to the time $t=0.15$ with $N=200$. Fig. 3 shows that AWENO-M scheme results in good resolution, in comparison with AWENO-JS and WENO schemes.

\subsubsection{Shock-density wave interaction case}

A shock wave of Mach number $M a=3$ interacts with a sinusoidal density field [38]. The initial conditions for pressure, velocity, and density fields are $[p, u, \rho]_{l}=$ $(31 / 3,4 \sqrt{35} / 9,27 / 7), x \in[-5,-4)$ and $[p, u, \rho]_{r}=(1,0,1+\alpha \sin (m x)), x \in[-4,5]$. Where $\alpha=0.2$ and $m=5.0$. The computations are performed up to time $t=1.8$ with CFL number 0.9 and $N=300$. The reference solution is evaluated using the mapped WENO scheme with $N=3000$. Fig. 4 shows that AWENO-M and WENO-M schemes capture fine-scale 


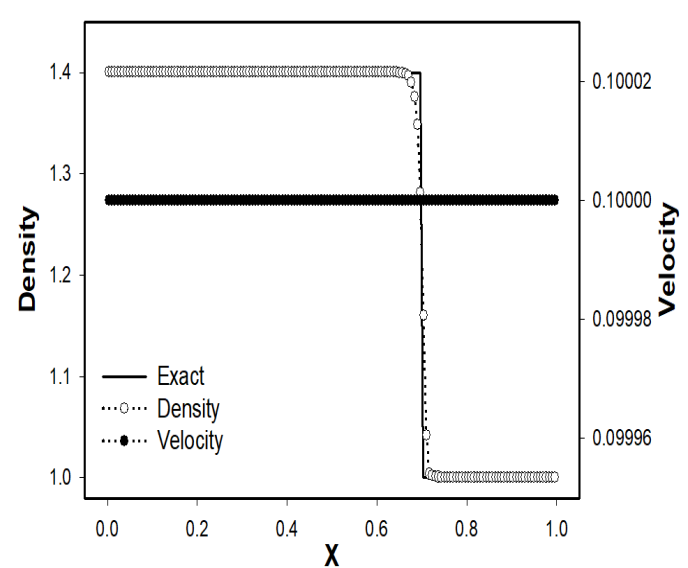

(a) Primitive variable reconstruction

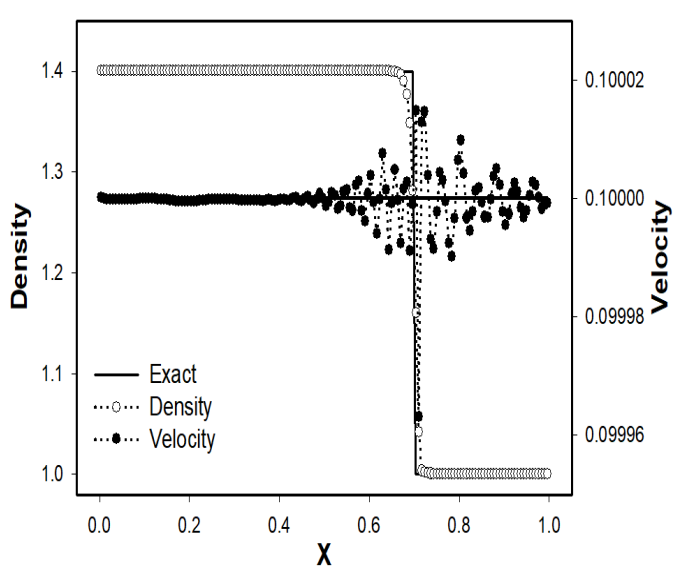

(b) Conservative variable reconstruction

Figure 2: The density and velocity profile of isolated contact discontinuity up to time $t=2$ with AWENO-M scheme ( $N=150$ grid points).

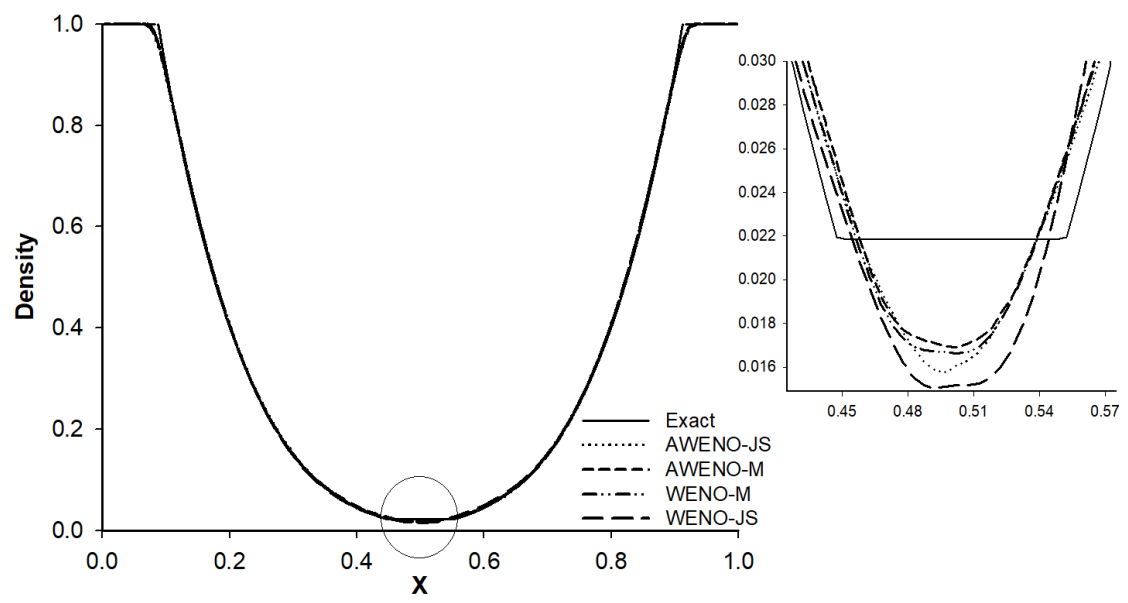

Figure 3: The density profile of the 123 problem with $N=200$ at final time $t=0.15$ (the enlarged view of the encircled part is shown on the right).

structures accurately as compare to AWENO-JS and WENO-JS schemes. Further, the present AWENO-M scheme is seen to better than the WENO-M scheme.

\subsubsection{Shock-entropy wave interaction case}

A shock wave of strength 1.1 interacts with a high-frequency oscillating density field [15]. The initial conditions for pressure, velocity, and density fields are

$$
(p, u, \rho)= \begin{cases}(1.5156,0.5233,1.8050), & x \in[-5.0,-4.5), \\ (1,0,1+\alpha \sin (m \pi x)), & x \in[-4.5,5],\end{cases}
$$



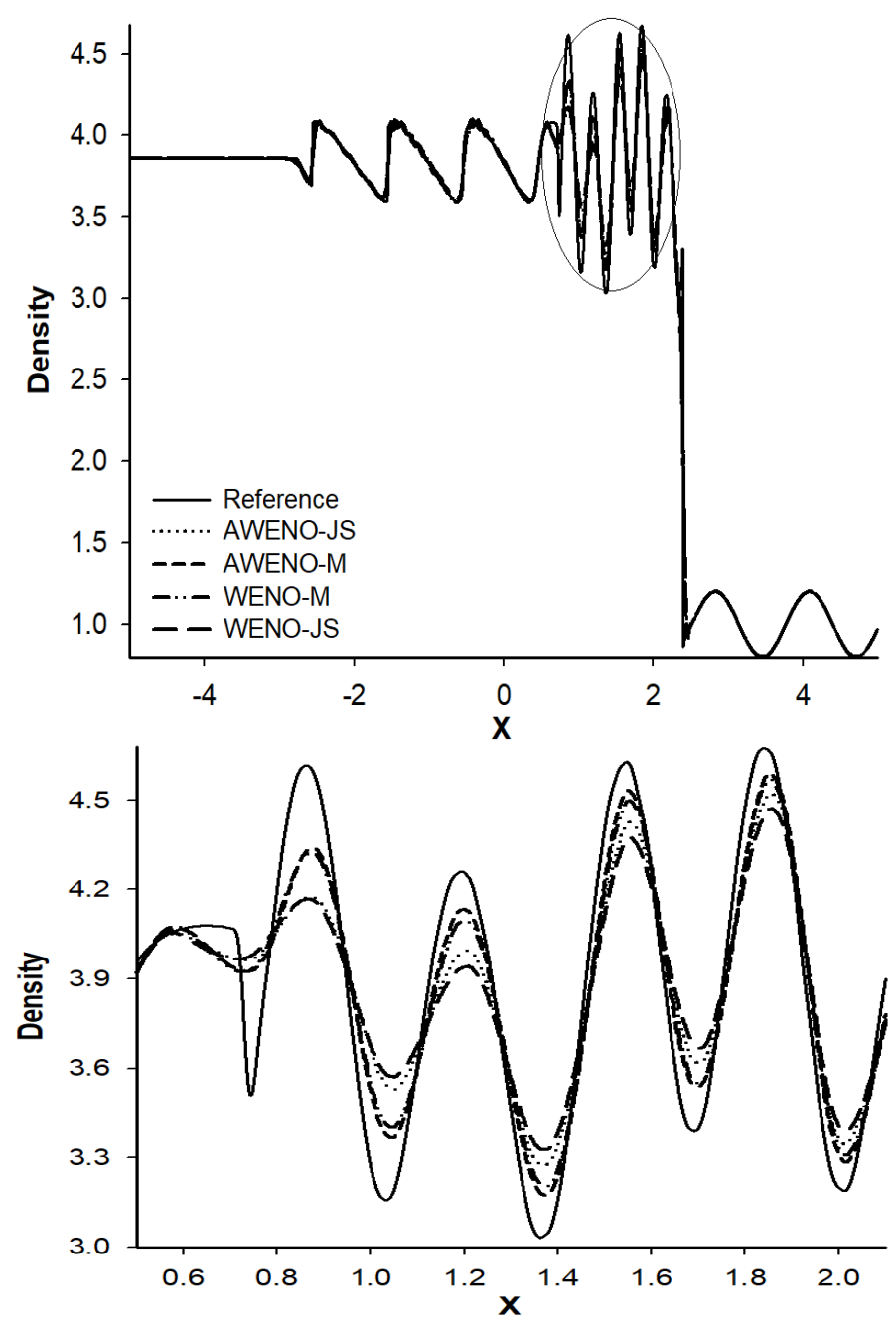

Figure 4: Results of shock-density interaction by AWENO and WENO schemes with $N=300$ grid points, full solution (top) and encircled part (bottom).

where $\alpha=0.1$ and $m=20$. Computations have been performed up to time $t=5.0$ with $N=$ 1500. The reference solution is evaluated by using the mapped WENO scheme with $N=$ 6000. The numerical results for the reference solution with three encircled regions (A, B, and $C$ ) across the full domain is shown in Fig. 5(a). The enlarged view of encircled regions are shown in Figs. 5(b), (c) and (d). We remark form Fig. 5 that the present AWENO$\mathrm{M}$ scheme resolves the flow structure more accurately as compared to the other highresolution schemes. 


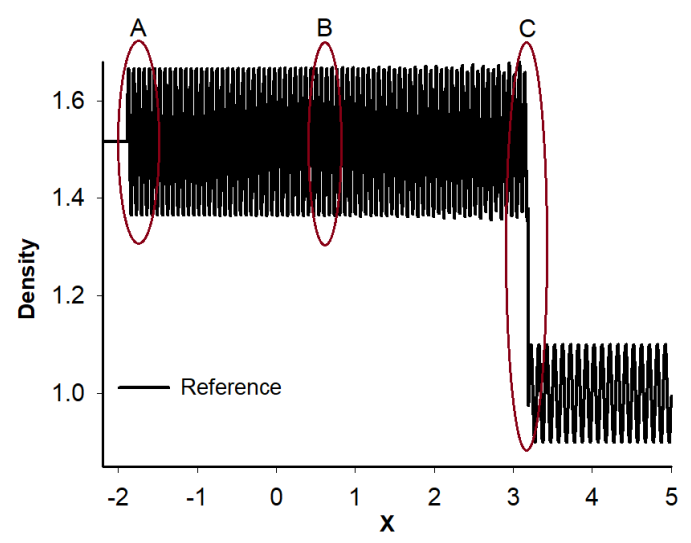

(a) Reference solution full domain

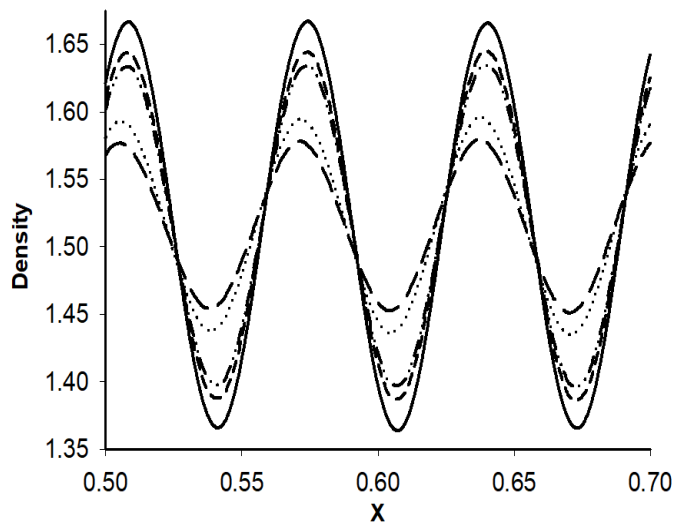

(c) Region B of full domain

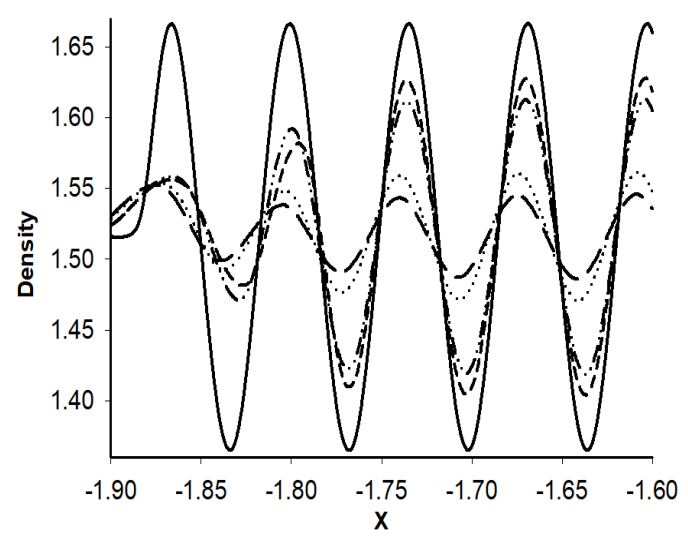

(b) Region A of full domain

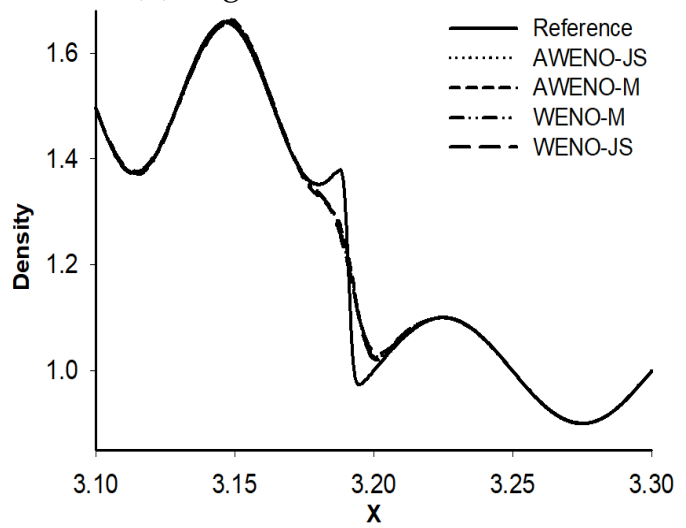

(d) Region C of full domain

Figure 5: Density profile of the shock-entropy interaction with $N=1500$ : (a) Reference solution full domain, and (b)-(d) the enlarged view of encircled region A, B, and C in full domain. Legends are shown in (d).

\subsubsection{Lax problem}

For the Riemann problem of Lax [39], the initial conditions for pressure, velocity, and density are $[p, u, \rho]_{l}=(3.528,0.698,0.445), x \in[0,0.5)$ and $[p, u, \rho]_{r}=(0.571,0,0.5), x \in[0.5,1)$. Numerical simulations have been performed up to the time $t=0.13$ with $N=200$. Fig. 6 shows that AWENO and WENO schemes result in the same level of accuracy compared to the exact solution.

\subsubsection{Sod's test case}

The modified Riemann problem of Sod's [40] is calculated up to time $t=0.2$ with various grid resolutions to demonstrate the convergence rate of WENO and AWENO schemes. The initial conditions for pressure, velocity, and density fields are $[p, u, \rho]_{l}=(1,0.75,1)$, $x \in[0,0.5)$ and $[p, u, \rho]_{r}=(0.1,0,0.125), x \in[0.5,1)$. From Table 4 , we can observe that WENO 


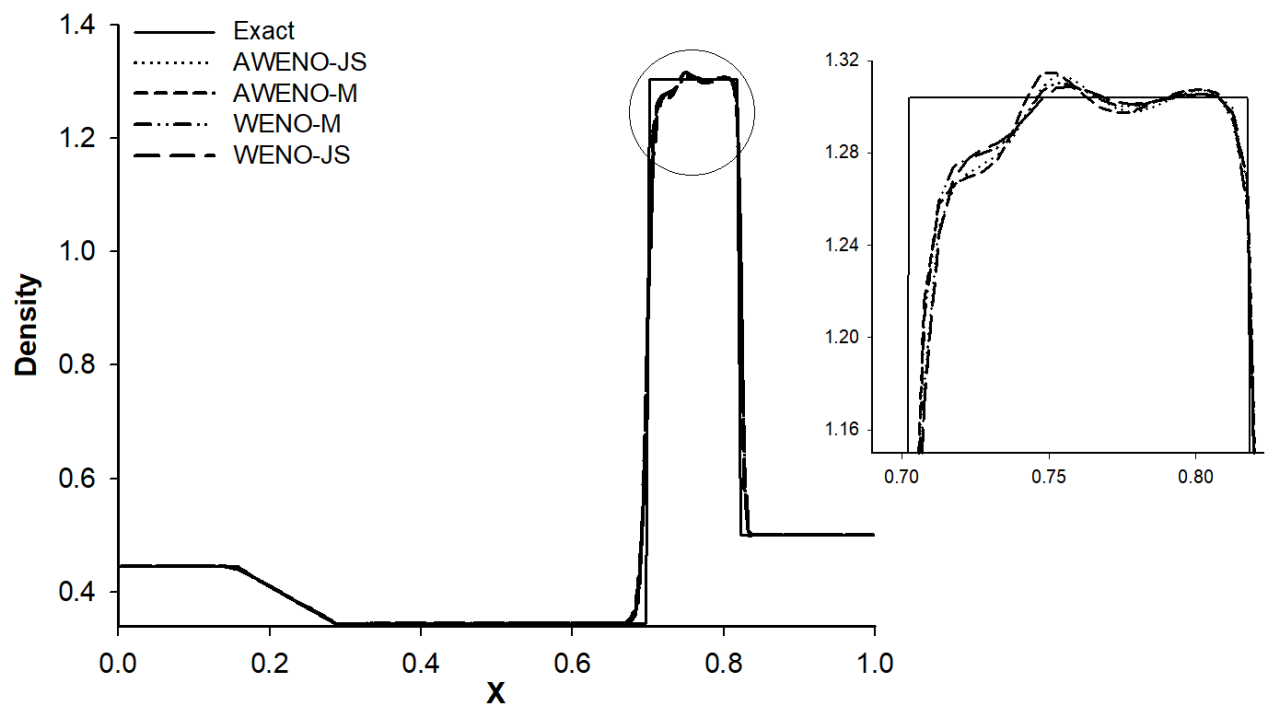

Figure 6: Density profile of the Lax problem with $N=200$ (the enlarged view of the encircled part is shown on the right).

Table 4: The $L_{1}$ error norm and $r_{c}$ of WENO and AWENO schemes, for modified Sod's test.

\begin{tabular}{||c|cccccccc||}
\hline \multirow{2}{*}{$N$} & \multicolumn{2}{|c}{ WENO-JS } & \multicolumn{2}{c}{ WENO-M } & \multicolumn{2}{c|}{ AWENO-JS } & \multicolumn{2}{c||}{ AWENO-M } \\
\cline { 2 - 9 } & $L_{1}$ & $r_{c}$ & $L_{1}$ & $r_{c}$ & $L_{1}$ & $r_{c}$ & $L_{1}$ & $r_{c}$ \\
\hline 80 & $7.6 e-3$ & - & $7.0 e-3$ & - & $7.3 e-3$ & - & $6.5 e-3$ & - \\
160 & $4.0 e-3$ & 0.92 & $3.7 e-3$ & 0.92 & $3.9 e-3$ & 0.90 & $3.4 e-3$ & 0.93 \\
320 & $2.2 e-3$ & 0.86 & $2.0 e-3$ & 0.86 & $2.1 e-3$ & 0.87 & $1.9 e-3$ & 0.87 \\
640 & $1.2 e-3$ & 0.89 & $1.1 e-3$ & 0.94 & $1.1 e-3$ & 0.94 & $9.2 e-4$ & 1.02 \\
1280 & $6.1 e-4$ & 0.96 & $5.3 e-4$ & 1.02 & $5.7 e-4$ & 0.97 & $4.5 e-4$ & 1.03 \\
2560 & $3.4 e-4$ & 0.86 & $2.8 e-4$ & 0.90 & $3.2 e-4$ & 0.84 & $2.4 e-4$ & 0.89 \\
5120 & $1.6 e-4$ & 1.04 & $1.3 e-4$ & 1.07 & $1.5 e-4$ & 1.03 & $1.1 e-4$ & 1.10 \\
10240 & $8.8 e-5$ & 0.91 & $7.2 e-5$ & 0.89 & $8.2 e-5$ & 0.91 & $6.0 e-5$ & 0.91 \\
20480 & $4.9 e-5$ & 0.84 & $3.8 e-5$ & 0.91 & $4.5 e-5$ & 0.86 & $3.3 e-5$ & 0.88 \\
40960 & $2.8 e-5$ & 0.82 & $2.1 e-5$ & 0.85 & $2.5 e-5$ & 0.83 & $1.8 e-5$ & 0.87 \\
81920 & $1.6 e-5$ & 0.82 & $1.2 e-5$ & 0.82 & $1.4 e-5$ & 0.82 & $1.0 e-5$ & 0.84 \\
163840 & $8.9 e-6$ & 0.81 & $6.8 e-6$ & 0.82 & $8.2 e-6$ & 0.81 & $5.7 e-6$ & 0.83 \\
\hline
\end{tabular}

and AWENO schemes show the same level of accuracy. The convergence rate $r_{c}$ is of the first order, which is the property of all shock capturing methods during the solution of hyperbolic equations with embedded discontinuities [24]. The proposed AWENO-M scheme results in a less $L_{1}$ error and a higher convergence rate compared to AWENO-JS and WENO schemes. 


\subsection{Two-dimensional problems}

In this subsection, the performance of the proposed AWENO-M scheme is analyzed for two-dimensional Euler problems, and results are compared with AWENO-JS and WENO schemes. We computed results for the gas dynamics (GD) problem, Rayleigh-Taylor instability (RTI), Kelvin-Helmholtz instability (KHI), and forward-facing step (FFS). Pressure $p$, velocity $\left(u_{1}, u_{2}\right)$, and density $\rho$ fields are used to describe initial condition. The reference solution is obtained using the AWENO-M scheme on a fine grid for comparing the numerical solution of high order schemes.

\subsubsection{Gas dynamics (GD) problem}

We perform simulation for two different configurations (GD-6 and GD-12) of the gas dynamics problems [41]. We have considered a square domain with zero gradient boundary conditions on all four sides. Initial pressure, velocity, and density for configuration 6 (GD-6) in a $2.0 \times 2.0$ domain are

$$
\left(p, u_{1}, u_{2}, \rho\right)=\left\{\begin{array}{lll}
(1.0,0.75,-0.5,1.0), & x_{1} \in[1.0,2.0], & x_{2} \in[1.0,2.0], \\
(1.0,0.75,0.5,2.0), & x_{1} \in[0.0,1.0], & x_{2} \in[1.0,2.0], \\
(1.0,-0.75,0.5,1.0), & x_{1} \in[0.0,1.0], & x_{2} \in[0.0,1.0], \\
(1.0,-0.75,-0.5,3.0), & x_{1} \in[1.0,2.0], & x_{2} \in[0.0,1.0] .
\end{array}\right.
$$

The solution to this problem consists of four slip lines. We compute the solution up to the final time $t=1.6$ with $800 \times 800$ grid points. A reference solution has been obtained with $1600 \times 1600$ mesh. The density contours are shown in Fig. 7. Comparison with reference solution Fig. 7(e) shows that the proposed AWENO-M scheme resolves vortical structures and shear instability better that AWENO-JS and WENO schemes. The initial conditions for configuration $12(\mathrm{GD}-12)$ in a $1.0 \times 1.0$ domain are

$$
\left(p, u_{1}, u_{2}, \rho\right)=\left\{\begin{array}{lll}
(0.4,0.0,0.0,0.5313), & x_{1} \in[0.5,1.0], & x_{2} \in[0.5,1.0], \\
(1.0,0.7276,0.0,1.0), & x_{1} \in[0.0,0.5], & x_{2} \in[0.5,1.0], \\
(1.0,0.0,0.0,0.8), & x_{1} \in[0.0,0.5], & x_{2} \in[0.0,0.5], \\
(1.0,0.0,0.7276,1.0), & x_{1} \in[0.5,1.0], & x_{2} \in[0.0,0.5] .
\end{array}\right.
$$

The solution of this problem includes two contact waves and two shock waves. The simulation has been performed with CFL number 0.8 up to time $t=0.25$ on a $600 \times 600$ mesh. For comparison, a reference solution has been obtained using a fine $(1200 \times 1200)$ mesh. Density contours in Fig. 8 with the AWENO-M scheme are closer to the reference solution in Fig. 8(e). Thus, AWENO-M resolves the fine-scale structures more accurately than AWENO-JS and WENO schemes.

\subsubsection{Kelvin-Helmholtz instability (KHI)}

It is a standard problem used for describing the dissipative behavior of high-resolution schemes [42]. Simulations are performed in a square domain of $1.0 \times 1.0$ with periodic 


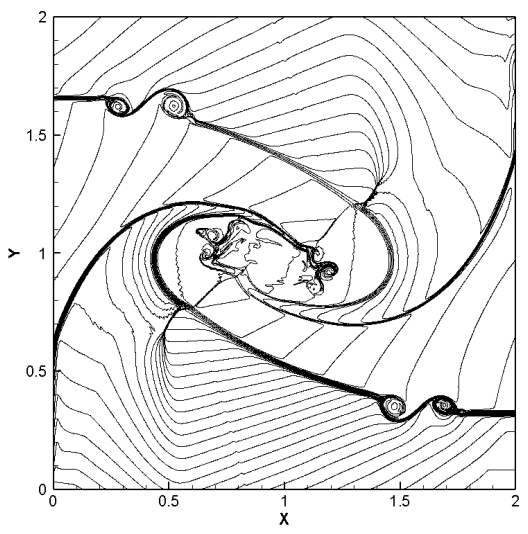

(a) WENO-JS

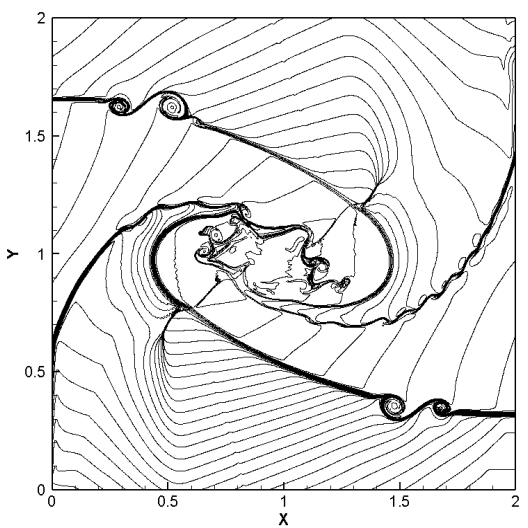

(c) AWENO-JS

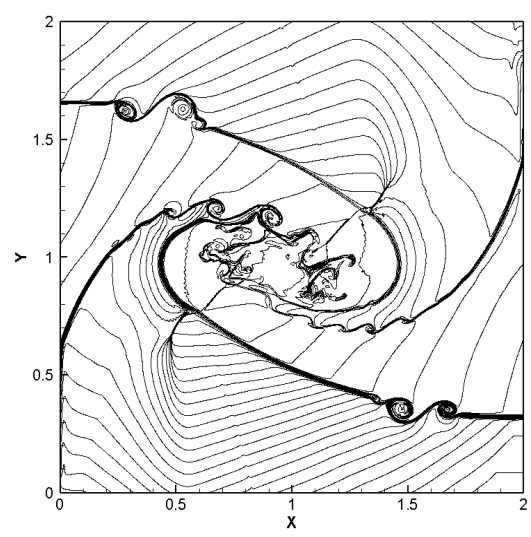

(b) WENO-M

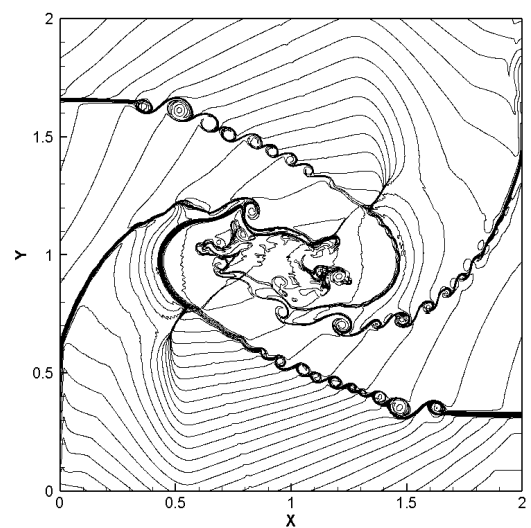

(d) AWENO-M

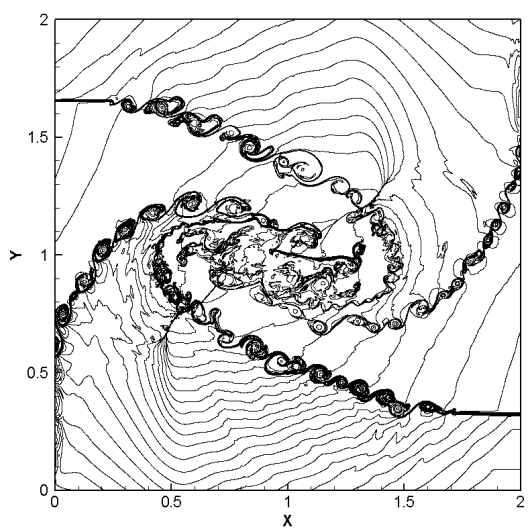

(e) Reference solution

Figure 7: Numerical results for gas dynamics problem. Density contours of configuration 6. 


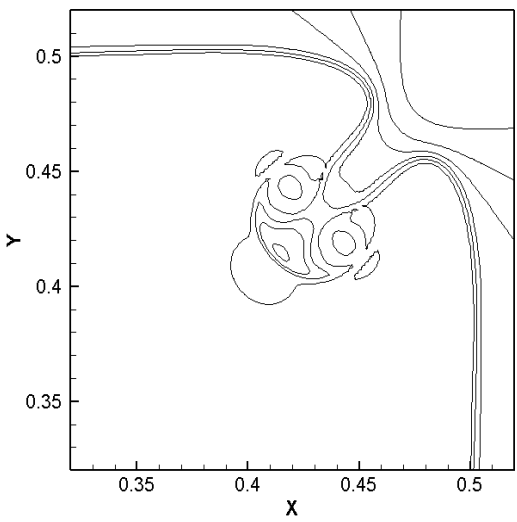

(a) WENO-JS

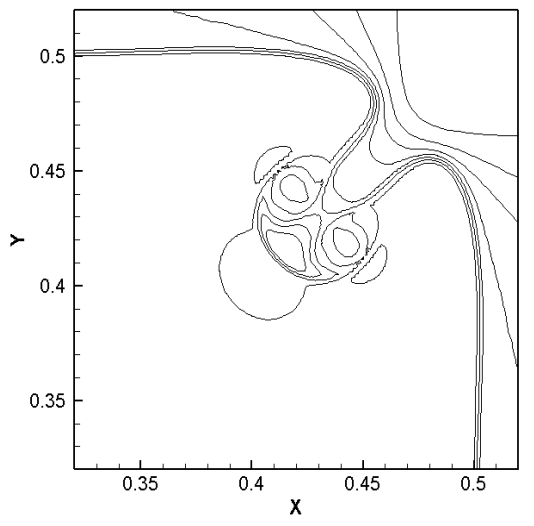

(c) AWENO-JS

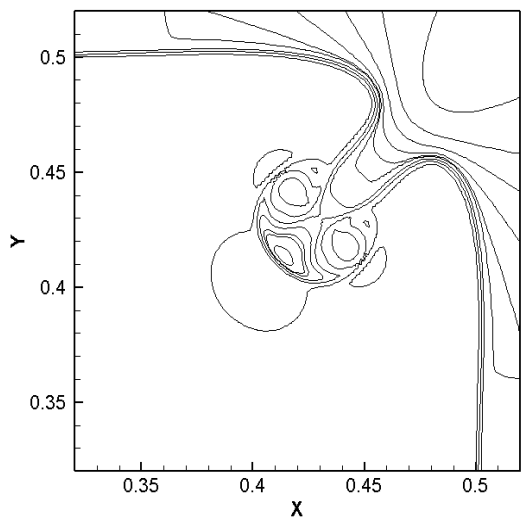

(b) WENO-M

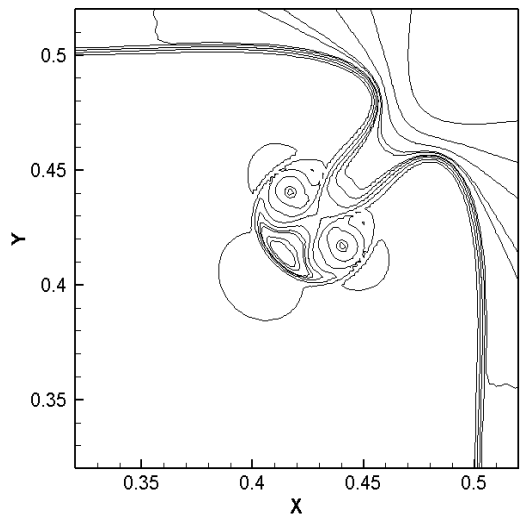

(d) AWENO-M

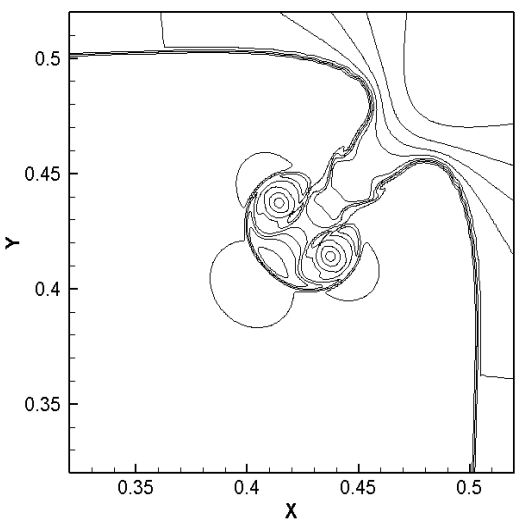

(e) Reference solution

Figure 8: Numerical results for gas dynamics problem. Density contours of configuration 12. 
boundary on a $1024 \times 1024$ grid up to time $t=1.0$. The initial conditions for pressure, velocity, and density are

$$
\left(p, u_{1}, u_{2}, \rho\right)=\left\{\begin{array}{lll}
\left(2.5,0.5, \alpha \sin \left(2 \pi x_{1} / l_{0}\right), 1.0\right), & x_{1} \in[-0.5,0.5], & x_{2} \in[-0.5,-0.25), \\
\left(2.5,-0.5, \alpha \sin \left(2 \pi x_{1} / l_{0}\right), 2.0\right), & x_{1} \in[-0.5,0.5], & x_{2} \in[-0.25,0.25], \\
\left(2.5,0.5, \alpha \sin \left(2 \pi x_{1} / l_{0}\right), 1.0\right), & x_{1} \in[-0.5,0.5], & x_{2} \in(0.25,0.5],
\end{array}\right.
$$

where $\alpha=0.1$ and $l_{0}=1.0$. Numerical results in Fig. 9 show that the AWENO-M scheme result is well-matched with the reference solution in Fig. 9(e). Therefore, the proposed AWENO-M scheme captures the complex structure with greater accuracy as compared to AWENO-JS and WENO schemes.

\subsubsection{Rayleigh-Taylor instability (RTI)}

In this test case, we assess the robustness of the high order scheme in the form of complex structures related to the numerical viscosity of the scheme [43]. We consider a rectangular domain $0.25 \times 1.0$ and solve till time $t=1.95$ with different grid resolutions. The initial conditions for pressure, velocity, and density are

$$
\left(p, u_{1}, u_{2}, \rho\right)= \begin{cases}\left(2 x_{2}+1,0.0,-0.025 \sqrt{\gamma p / \rho} \cos \left(8 \pi x_{1}\right), 2.0\right), & x_{2} \in[0.0,0.5], \\ \left(2 x_{2}+3 / 4,0.0,-0.025 \sqrt{\gamma p / \rho} \cos \left(8 \pi x_{1}\right), 1.0\right), & x_{2} \in[0.5,1.0],\end{cases}
$$

where $\gamma=1.67$ is the ratio of specific heat. Reflecting boundary conditions are applied to the left (inlet) and right (outlet) boundary. The bottom and top boundary values are set as $p=1.0, u_{1}=u_{2}=0.0, \rho=2.0$ and $p=2.5, u_{1}=u_{2}=0.0, \rho=1.0$, respectively. The source term density $\rho$ is added to the $x_{2}$-momentum equation, and the product $\rho u_{2}$ is added to the energy equation. Density contours in Fig. 10 with the proposed AWENO-M scheme are similar to the reference solution in Fig. 10(e). Thus, the AWENO-M resolves the small scale structures and roll-ups more accurately as compared to AWENO-JS and WENO schemes.

\subsubsection{Forward facing step (FFS)}

We consider a uniform Mach 3 flow on a rectangular domain of $3.0 \times 1.0$ with a forwardfacing step size of $[0.0,0.6] \times[0.0,0.2]$ [44]. Computations are performed on a $960 \times 320$ grid up to final time $t=4.0$. The initial conditions are $\left(p, u_{1}, u_{2}, \rho\right)=(1.0,3.0,0.0,1.4)$ and specific heat ratio, $\gamma=1.4$. Gas with this pressure, velocity, and density enters the inlet boundary. The reflecting boundary condition is applied on the wall, and supersonic outflow is prescribed on the right boundary. The density contours in Fig. 11 with the AWENO-M scheme are closer to the reference solution in Fig. 11(e). The AWENO-M scheme captures more numbers of roll-ups of vortices in the shear layer region with high resolution compared to AWENO-JS and WENO schemes. 


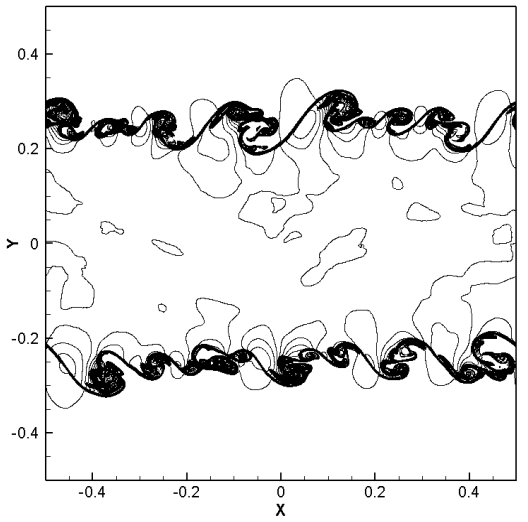

(a) WENO-JS

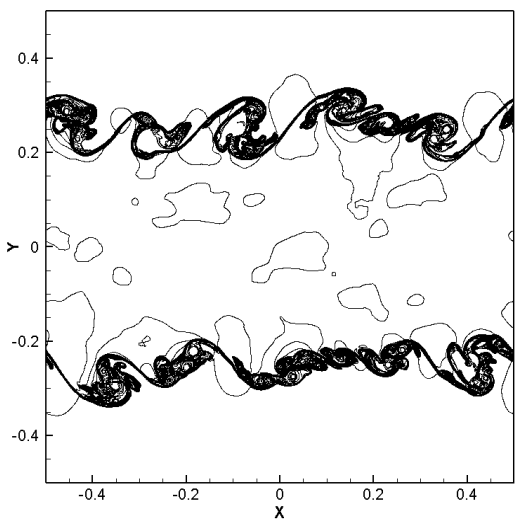

(c) AWENO-JS

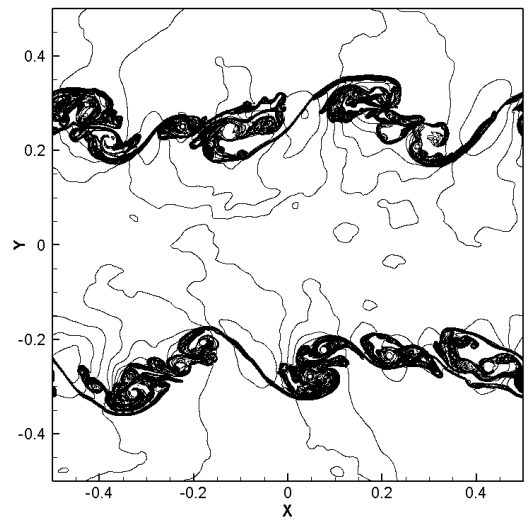

(b) WENO-M

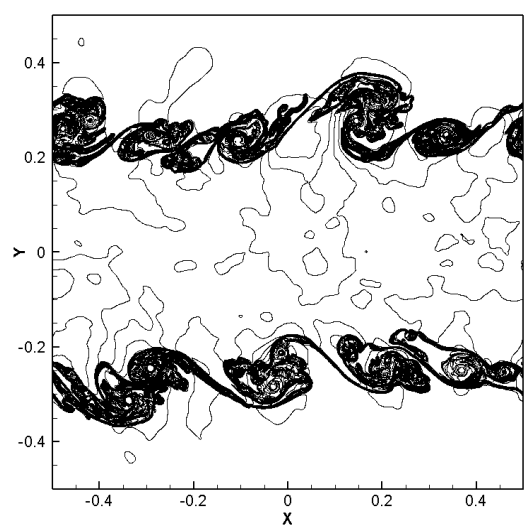

(d) AWENO-M

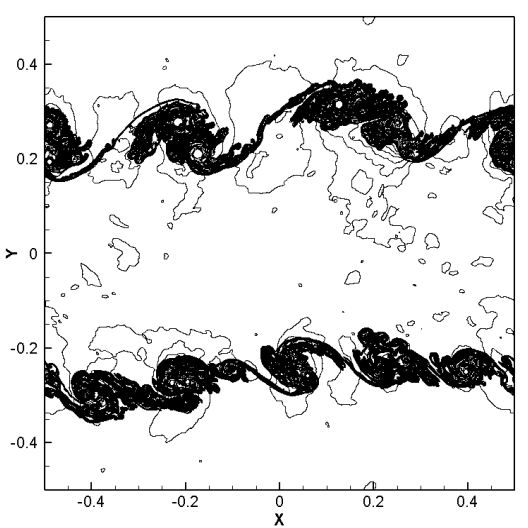

(e) Reference

Figure 9: Density contours for Kelvin-Helmholtz instability problem: (a)-(d) $1024 \times 1024$ grid, and (e) reference solution with $2048 \times 2048$ mesh. 


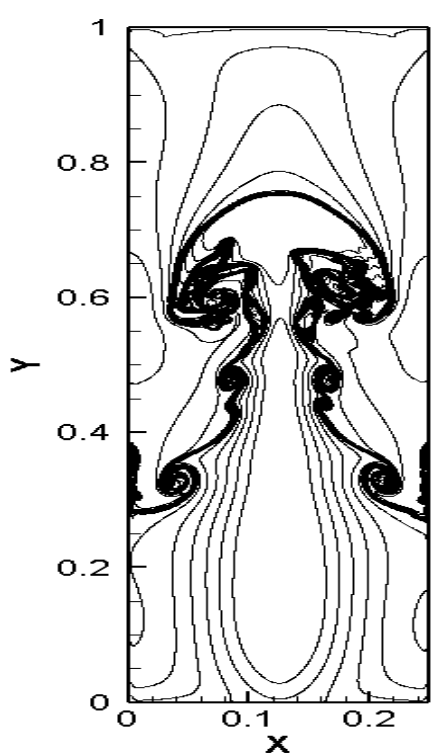

(a) WENO-JS

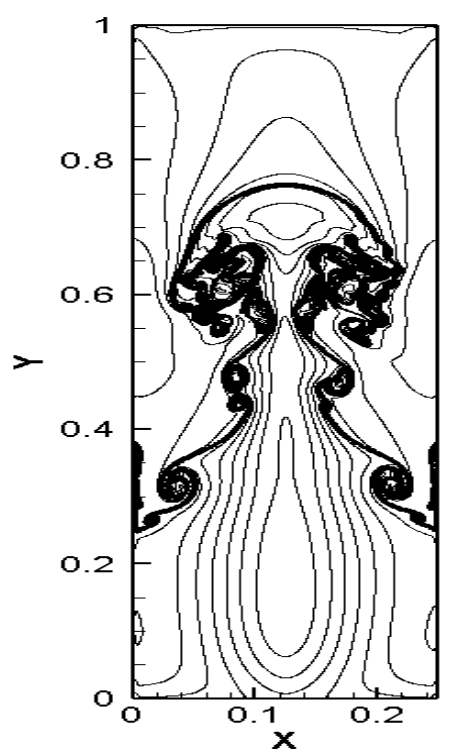

(b) WENO-M

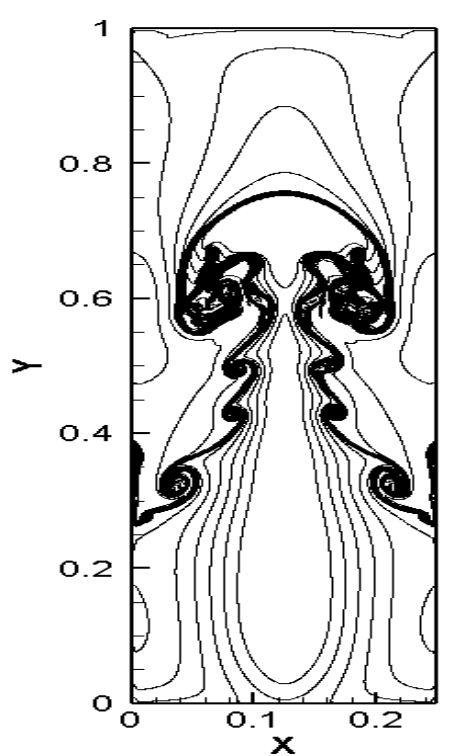

(c) AWENO-JS

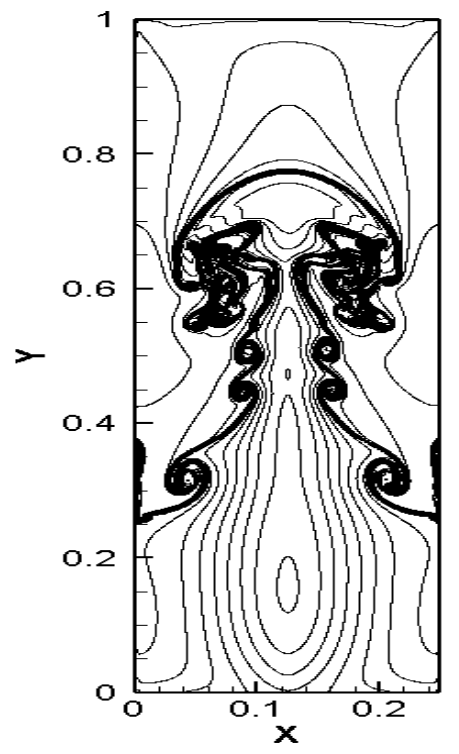

(d) AWENO-M

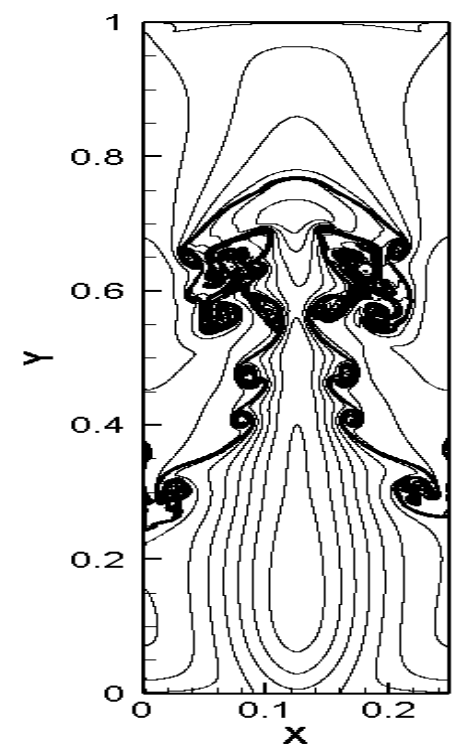

(e) Reference

Figure 10: Density profile for Rayleigh-Taylor instability: (a)-(d) with $240 \times 960$ grid, and (e) reference solution with $480 \times 1920$ grid.

\subsubsection{Comparison of computational efficiency}

In Table 5, we show the computational time (or CPU time) for GD-6, GD-12, KHI, RTI, and FFS problems with different mesh resolutions to understand the computational efficiency 


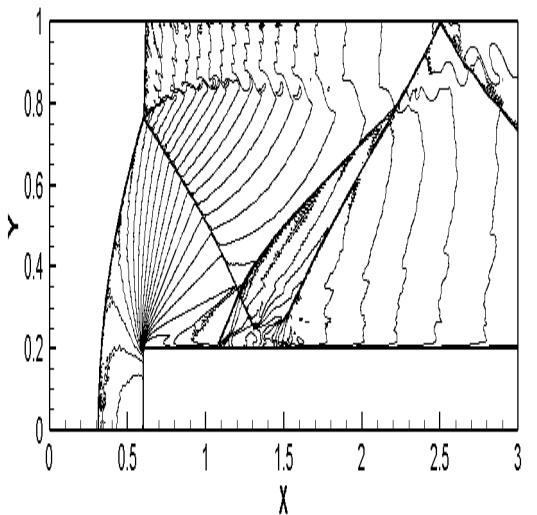

(a) WENO-JS

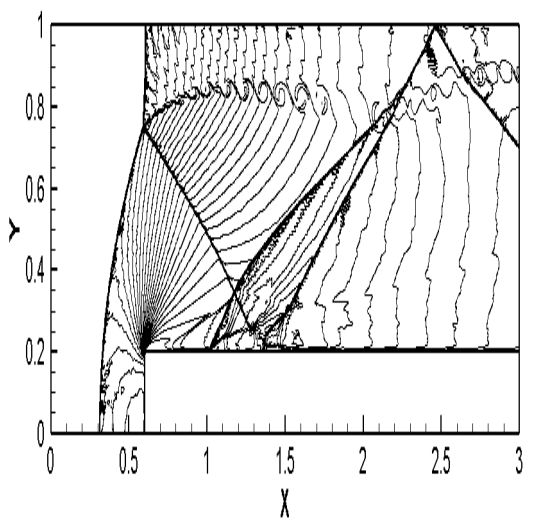

(c) AWENO-JS

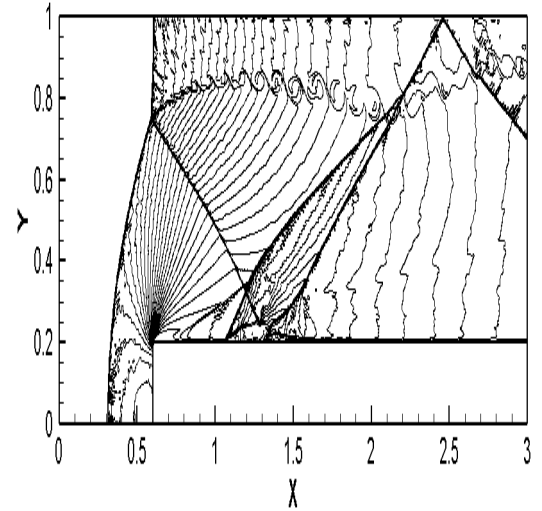

(b) WENO-M

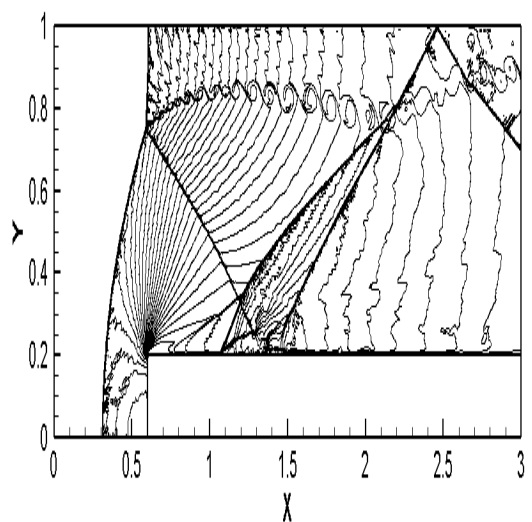

(d) AWENO-M

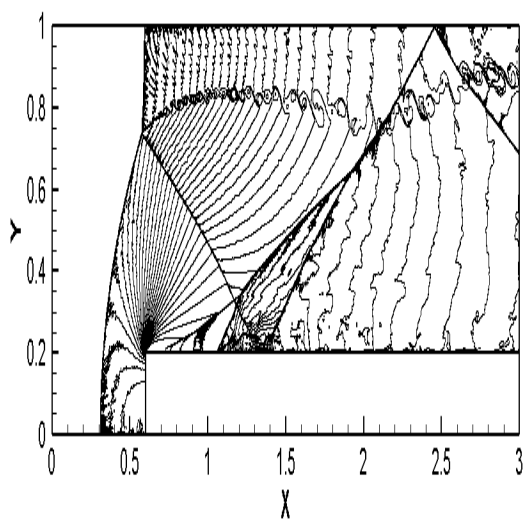

(e) Reference solution

Figure 11: Density contours for forward facing step. A $1920 \times 640$ mesh has been used for the reference solution. 
Table 5: The computation time (in seconds) for the high order schemes (WENO and AWENO) on various mesh resolutions. The bracketed number (ratio) is the ratio of computation time between WENO-JS and the other scheme.

\begin{tabular}{||cccccc||}
\hline & Resolution & WENO-JS & WENO-M & AWENO-JS & AWENO-M \\
\hline \multirow{3}{*}{ GD-6 } & $200 \times 200$ & 120 & $123(1.02)$ & $136(1.13)$ & $134(1.12)$ \\
& $400 \times 400$ & 965 & $1003(1.04)$ & $1095(1.14)$ & $1086(1.13)$ \\
& $800 \times 800$ & 8186 & $8547(1.04)$ & $9498(1.16)$ & $9426(1.15)$ \\
GD-12 & $150 \times 150$ & 18 & $18(1.02)$ & $20(1.12)$ & $20(1.11)$ \\
& $300 \times 300$ & 148 & $153(1.03)$ & $169(1.14)$ & $167(1.13)$ \\
& $600 \times 600$ & 1192 & $1241(1.04)$ & $1372(1.15)$ & $1360(1.14)$ \\
KH & $256 \times 256$ & 376 & $386(1.03)$ & $421(1.12)$ & $417(1.11)$ \\
& $1024 \times 512$ & 3136 & $3278(1.05)$ & $3610(1.15)$ & $3578(1.14)$ \\
& $60 \times 240$ & 127 & $131(1.03)$ & $141(1.11)$ & $140(1.11)$ \\
RTI & $120 \times 480$ & 1006 & $1038(1.03)$ & $1141(1.13)$ & $1130(1.12)$ \\
& $240 \times 960$ & 7893 & $8210(1.04)$ & $9009(1.14)$ & $8971(1.14)$ \\
& $240 \times 80$ & 196 & $202(1.03)$ & $222(1.13)$ & $220(1.12)$ \\
FFS & $480 \times 160$ & 1451 & $1511(1.04)$ & $1672(1.15)$ & $1655(1.14)$ \\
& $960 \times 320$ & 11573 & $12108(1.05)$ & $13451(1.16)$ & $13333(1.15)$ \\
\hline
\end{tabular}

of WENO and AWENO schemes. The results show that the proposed AWENO-M scheme takes $1 \%$ less CPU time for all test problems than the AWENO-JS scheme.

\section{Conclusions}

We have designed a fifth order alternative mapped WENO finite volume scheme (AWENO-M) for solving non-linear hyperbolic equations in this work. The AWENO$M$ scheme utilizes non-linear weights of mapped WENO scheme (WENO-M) [24]. In the present scheme, numerical flux is evaluated using the reconstruction of primitive variables rather than conservative variables. The current method exhibits the optimal (fifth-order) rate of convergence at critical points. It results in less diffusion for onedimensional test cases as compared to the traditional WENO scheme (WENO-JS) [8], WENO-M, and alternative WENO scheme based on WENO-JS non-linear weights [19] (AWENO-JS). For two-dimensional problems, the proposed scheme resolves the finescale structure accurately. Further, it captures the flow structures across the shear lines better than AWENO-JS and WENO schemes.

\section{Acknowledgements}

Authors gratefully acknowledge the computational facilities of CFD Lab., MIED, IITRoorkee established with the FIST grant (DST-354-MID) from DST, Government of India. 


\section{References}

[1] C. W. SHU, Essentially non-oscillatory and weighted essentially non-oscillatory schemes for hyperbolic conservation laws, Tech. rep., NASA Langley Research Center, Hampton, 1997.

[2] C. W. SHU, High order weighted essentially nonoscillatory schemes for convection dominated problems, SIAM Rev., 51 (2009), pp. 82-126.

[3] A. HARTEN, AND S. OSHER, Uniformly high-order accurate non-oscillatory schemes, I, SIAM J. Numer. Anal., 24 (1987), pp. 279-309.

[4] A. HARTEN, B. ENGQUist, S. OSher, AND S. R. CHAKRAVARTHY, Uniformly high order accurate essentially non-oscillatory schemes, III, J. Comput. Phys., 131 (1987), pp. 3-47.

[5] A. HARTEN, High resolution schemes for hyperbolic conservation laws, J. Comput. Phys., 49 (1983), pp. 353-357.

[6] J. CASPER, AND H. L. ATKINS, A finite-volume high-order ENO scheme for two-dimensional hyperbolic systems, J. Comput. Phys., 106 (1993), pp. 62-76.

[7] X. D. LIU, S. OsHeR, AND T. CHAN, Weighted essentially non-oscillatory schemes, J. Comput. Phys., 115 (1994), pp. 200-212.

[8] G. S. JIANG, AND C. W. SHU, Efficient implementation of weighted ENO schemes, J. Comput. Phys., 228 (1996), pp. 202-228.

[9] D. S. BALSARA, AND C. W. SHU, Monotonicity preserving weighted essentially non-oscillatory schemes with increasingly high order of accuracy, J. Comput. Phys., 160 (2000), pp. 405-452.

[10] J. A. EKATERINARIS, High-order accurate, low numerical diffusion methods for aerodynamics, Progr. Aerosp. Sci., 41 (2005), pp. 192-300.

[11] Q. WANG, AND Y. X. REN, An accurate and robust finite volume scheme based on the spline interpolation for solving the Euler and Navier-Stokes equations on non-uniform curvilinear grids, J. Comput. Phys., 284 (2015), pp. 648-667.

[12] M. A. SHADAB, D. BALSARA, W. SHYY, AND K. XU, Fifth order finite volume WENO in general orthogonally-curvilinear coordinates, Comput. Fluids, 190 (2019), pp. 398-424.

[13] W. F. HUANG, Y. X. REN, AND X. JIANG, A simple algorithm to improve the performance of the WENO scheme on non-uniform grids, Acta Mech. Sin., 34 (2018), pp. 37-47.

[14] Y. GUO, L. TANG, H. ZHANG, AND S. SONG, A maximum-principle-preserving third order finite volume SWENO scheme on unstructured triangular meshes, Adv. Appl. Math. Mech., 10 (2018), pp. 114-137.

[15] V. A. TitAREV, AND E. F. TORO, Finite-volume WENO schemes for three-dimensional conservation laws, J. Comput. Phys., 201 (2004), pp. 238-260.

[16] J. ZHU, AND J. QIU, A new type of finite volume WENO Schemes for hyperbolic conservation laws, J. Sci. Comput., 73 (2017), pp. 1338-1359.

[17] H. DONG, C. LU, AND H. YANG, The finite volume WENO with Lax-Wendroff scheme for nonlinear system of Euler equations, Mathematics, 6 (2018), pp. 1-17.

[18] C. W. SHU, AND S. OSHER, Efficient implementation of essentially non-oscillatory shock-capturing schemes, J. Comput. Phys., 77 (1988), pp. 439-471.

[19] Y. JIANG, C. W. SHU, AND M. ZHANG, An alternative formulation of finite difference weighted ENO schemes with Lax-Wendroff time discretization for conservation laws, SIAM J. Sci. Comput., 35 (2013), pp. 1137-1160.

[20] Y. JIANG, C. W. SHU, AND M. ZHANG, Free-stream preserving finite difference schemes on Curvilinear Meshes, Methods Appl. Anal., 21 (2014), pp. 1-30.

[21] H. LIU, A numerical study of the performance of alternative weighted ENO methods based on various numerical fluxes for conservation law, Appl. Math. Comput., 296 (2017), pp. 182-197. 
[22] Y. SHI, AND Y. GUO, A fifth order alternative compact-WENO finite difference scheme for compressible Euler equations, J. Comput. Phys., 397 (2019), 108873.

[23] H. LIU, AND J. QIU, Finite difference Hermite WENO schemes for conservation laws, II: An alternative approach, J. Sci. Comput., 66 (2016), pp. 598-624.

[24] A. K. Henrick, T. D. Aslam, AND J. M. POWERs, Mapped weighted essentially non-oscillatory schemes: Achieving optimal order near critical points, J. Comput. Phys.,207 (2005), pp. 542-567.

[25] R. Borges, M. CARMONA, B. COSTA, AND W. S. DON, An improved weighted essentially nonoscillatory scheme for hyperbolic conservation laws, J. Comput. Phys., 227 (2008), pp. 3191-3211.

[26] M. CASTRO, B. COSTA, AND W. S. DON, High order weighted essentially non-oscillatory WENOZ schemes for hyperbolic conservation laws, J. Comput. Phys., 230 (2011), pp. 1766-1792.

[27] B. S. WANG, P. LI, Z. GAO, AND W. S. DON, An improved fifth order alternative WENO-Z finite difference scheme for hyperbolic conservation laws, J. Comput. Phys., 374 (2018), pp. 469-477.

[28] Z. GAO, L.-L. FANG, B.-S. WANG, Y. WANG, AND W. DON, Seventh and ninth orders characteristic-wise alternative weno finite difference schemes for hyperbolic conservation laws, Comput. Fluids, 202 (2020), 104519.

[29] P. LI, W. DON, AND Z. GAO, High order well-balanced finite difference WENO interpolationbased schemes for shallow water equations, Comput. Fluids, 201 (2020), 104476.

[30] W. DON, D.-M. LI, Z. GAO, AND B.-S. WANG, A characteristic-wise alternative WENO-Z finite difference scheme for solving the compressible multicomponent non-reactive flows in the overestimated quasi-conservative form, J. Sci. Comput., 82 (2020), pp. 1-24.

[31] Y. YU, S. XU, M. ZHANG, AND Z. ZHANG, Computation on premixed combustion of methane and air mixture induced by cylinders with different radiuses, Acta Aerodyn. Sin., 38 (2020), pp. $35-42$.

[32] L. XU, Q. WU, P. WeNG, AND B. ZHANG, HLLC Riemann solver based on high-order reconstruction for unsteady inviscid compressible flows, 2011 IEEE Int. Conf. Comput. Sci. Autom. Eng., Shanghai, 2011, pp. 618-622.

[33] U. S. RAJPUT, AND K. M. SINGH, Numerical experiments of flux difference splitting methods with high resolution scheme for supersonic flows, J. Phys. Conf. Ser., 1240 (2019), pp. 1-9.

[34] E. F. TORO, Riemann Solvers and Numerical Methods for Fluid Dynamics, 3rd Edition, Springer, Heidelberg, 2009.

[35] E. F. TORO, M. Spruce, and W. Speares, Restoration of the contact surface in the HLL-Riemann solver, Shock Waves, 4 (1994), pp. 25-34.

[36] C. W. SHU, Total variation diminishing time discretizations, SIAM J. Sci. Statist. Comput., 9 (1988), pp. 1073-1084.

[37] P. Batten, M. A. Leschziner, And U. C. Goldberg, Average state Jacobians and implicit methods for compressible viscous and turbulent flows, J. Comput. Phys., 137 (1997), pp. 38-78.

[38] C. W. SHU, AND S. OsHER, Efficient implementation of essentially non-oscillatory shock-capturing schemes, II, J. Comput. Phys., 83 (1989), pp. 32-78.

[39] P. D. LAX, Weak solutions of nonlinear hyperbolic equations and their numerical computation, Commun. Pure Appl. Math., 7 (1954), pp. 159-193.

[40] G. A. SOD, A survey of several finite difference methods for systems of nonlinear hyperbolic conservation laws, J. Comput. Phys., 27 (1978), pp. 1-31.

[41] C. W. SChUlZ-RinNE, J. P. Collins, and H. M. Glaz, Numerical solution of the Riemann problem for two-dimensional gas dynamics, SIAM J. Sci. Comput., 14 (1993), pp. 1394-1414.

[42] O. SAN, AND K. KARA, Evaluation of Riemann flux solvers for WENO reconstruction schemes: Kelvin-Helmholtz instability, Comput. Fluids, 117 (2015), pp. 24-41.

[43] X. WU, AND Y. ZHAO, A high-resolution hybrid scheme for hyperbolic conservation laws, Int. J. 
Numer. Methods Fluids, 78 (2015), pp. 162-187.

[44] P. WOODWARD, AND P. COLELLA, The numerical simulation of two-dimensional fluid flow with strong shocks, J. Comput. Phys., 54 (1984), pp. 115-173. 